\section{§8. Velocity-Space Structures of Distribution Function in Toroidal Ion Temperature Gradient Turbulence}

\section{W atanabe, T.-H., Sugama, $H$.}

We have studied detailed velocity-space structures of ion distribution functions and related entropy balance in the toroidal ion temperature gradient (ITG) turbulence with the anomalous ion thermal transport, by means of the newly developed toroidal gyrokinetic-Vlasov simulation code for the flux tube geometry [1]. The kinetic simulations with high velocity-space resolution enable us to quantitatively discuss the entropy balance which has rarely been evaluated in conventional studies.

Simulation results of the the toroidal ITG turbulent transport clearly show generation of the fine velocity-space structures of the distribution function and their collisional dissipation. Detailed calculation of the entropy balance confirms the statistically steady state of turbulence, where the anomalous transport balances with the dissipation given by the weak collisionality. The above results obtained by simulations with high velocity-space resolution are also understood in terms of generation, transfer, and dissipation processes of the entropy variable in the phase space.

The statistically steady toroidal ITG turbulence is observed in terms of the saturated states of the entropy variable, the potential energy, the ion heat transport flux, and the collisional dissipation. Detailed calculation of the entropy balance for the toroidal flux tube geometry confirms the steady state of the turbulence where the transport balances with the collisional dissipation in the same way as found in the slab ITG system [2]. Fine velocity-space structures of the perturbed ion distribution function clearly appear in fluctuations with large wavenumbers as shown in Fig.1, while the heat transport flux is mainly produced by vortices with long wavelengths. Accordingly, the entropy variable produced by the unstable modes with a macro velocity-scale is transferred in the wave number and velocity spaces, and is finally dissipated in the micro scale by the finite collision. Thus, the statistically steady toroidal ITG turbulence can be sustained, as we have seen in the slab case.

Introduction of the finite collisionality also makes the long-time simulation of the turbulent transport possible by smoothing out the fine-scale structures of the distribution function. According to our

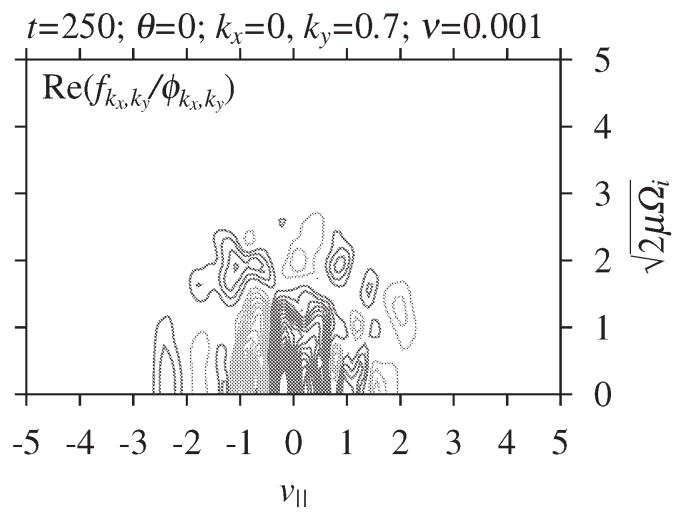

Figure 1: Velocity-space profile of real part of the perturbed distribution function observed in the toroidal ITG turbulence simulation at $t=$ $250 L_{n} / v_{t i}$ for $k_{y}=0.7 \rho_{i}^{-1}$ where $\theta=z=0$ and $k_{x}=0$. The horizontal and vertical axes are defined by $v_{\|}$and $\sqrt{2 \mu \Omega_{i}}$, respectively.

previous studies [2] on the slab ITG turbulence, the collision frequency used in the present study $\left(\nu_{i i}=10^{-3} v_{t i} / L_{n}\right)$ is in a regime where the transport flux has a logarithmic dependence on $\nu_{i i}$. Even if one employs numerically-enhanced diffusivity instead of the collision term with coarser phase-space resolution so as to carry out a collisionless turbulence simulation for the same parameters as used here, the transport coefficient may deviate from that in the true collisionless limit. Therefore, in order to quantitatively study the collisionality dependence of the velocity-space structures of the distribution function, one needs to perform numerical simulations with higher phase-space resolution for a lower collision frequency, which remains for future works.

\section{References}

[1] T.-H. Watanabe and H. Sugama, in "Proceedings of the 20th IAEA Fusion Energy Conference" (IAEA, Vienna, 2005).

[2] Watanabe, T.-H., Sugama, H., Phys. Plasmas 11, 1476 (2004). 